\title{
Automatic modulation classification of digital modulations in presence of HF noise
}

\author{
Hazza Alharbi ${ }^{3 *}$, Shoaib Mobien ${ }^{2}$, Saleh Alshebeili ${ }^{1,2,3}$ and Fahd Alturki ${ }^{3}$
}

\begin{abstract}
Designing an automatic modulation classifier (AMC) for high frequency (HF) band is a research challenge. This is due to the recent observation that noise distribution in $\mathrm{HF}$ band is changing over time. Existing AMCs are often designed for one type of noise distribution, e.g., additive white Gaussian noise. This means their performance is severely compromised in the presence of $\mathrm{HF}$ noise. Therefore, an AMC capable of mitigating the time-varying nature of HF noise is required. This article presents a robust AMC method for the classification of FSK, PSK, OQPSK, QAM, and amplitude-phase shift keying modulations in presence of HF noise using feature-based methods. Here, extracted features are insensitive to symbol synchronization and carrier frequency and phase offsets. The proposed AMC method is simple to implement as it uses decision-tree approach with pre-computed thresholds for signal classification. In addition, it is capable to classify type and order of modulation in both Gaussian and non-Gaussian environments.
\end{abstract}

Keywords: Automatic modulation classification, Feature-based classification, Bi-kappa noise, HF communications

\section{Introduction}

State-of-the-art digital communications and signal processing techniques have caused a major resurgence in high frequency (HF) communication systems by reducing equipment size, improving communication reliability, and shortening the deployment time. Therefore, these systems provide a cost effective and versatile solution for long-haul communications which can be used in a wide variety of civilian and military scenarios, e.g., nation-wide emergency communication during natural disaster, war-zone communications, electronic surveillance, remote area monitoring, etc. Numerous scenariospecific standardized waveforms exist for HF radios depending on the required data rate and available transmit bandwidth [1]. An HF radio capable of communicating using all the standardized waveforms is desirable as it brings interoperability, flexibility, and cost efficiency. A software-defined radio (SDR) allows an HF radio to communicate using multiple standards. An automatic modulation classifier (AMC) is an essential part of a multi-standard communication system as it

\footnotetext{
*Correspondence: hazza.ksa@gmail.com

${ }^{3}$ Electrical Engineering Department, King Saud University, Riyadh, Saudi Arabia

Full list of author information is available at the end of the article
}

allows blind detection of the modulation schemes present in the received signal. However, designing an AMC for HF systems is not a trivial task, as the transmit signal is severely distorted due to ionospheric effects, multipath propagation, and non-Gaussian timevarying noise [2-4]. The non-Gaussian and time-varying nature of HF noise is a recent observation [3]. It has been noted in [4] that the HF noise follows Gaussian (G) or Bi-kappa (BK) distributions depending on day time. A BK distribution function is defined mathematically as [3]

$$
p(x, k)= \begin{cases}\frac{1}{2 \sqrt{\pi \sigma}}\left[1+\frac{x^{2}}{k \sigma^{2}}\right]^{-k} & k>0 \\ \frac{1}{2 \sqrt{\pi \sigma}} & k=0 \\ \frac{1}{2 \sqrt{\pi \sigma}}\left[1-\frac{x^{2}}{k \sigma^{2}}\right]^{k} & k<0\end{cases}
$$

where $\sigma$ and $k$ are the shaping parameter and tuning factor, respectively. Practical values of these parameters are $\sigma=46, k=1.1$ and $\sigma=20, k=1$ [3].

Till now, designing AMC algorithms in presence of non-Gaussian noise has not received sufficient attention. The reason is that Gaussianity assumption often matches the observed statistical characteristics of channel noise. 
However, for HF channel the case is different as the noise is better described by a model fluctuating between $G$ and BK distributions. Therefore, this new noise model must be considered during the development of an AMC for $\mathrm{HF}$ band. Existing AMC methods (that assume $\mathrm{G}$ noise only) are grouped into two categories: likelihood based (LB) and feature based (FB) methods. LB methods have two steps: calculating the likelihood function of the received signal for all candidate modulations, and then using maximum likelihood ratio test for decision-making [5]. In FB methods, features are first extracted from the received signal and then applied to a classifier in order to recognize the modulation type and possibly its order.

Figure 1 shows the block diagram of FB methods in an SDR. The front-end of SDR is the signal detection block which identifies a signal transmission [6]. This block is followed by an AMC which contains a feature extractor and a classifier.An overwhelming number of proposed features exist in AMC literature. Some of the most popular features include instantaneous amplitude, phase, and frequency [7-9], statistical features such as higher order moments and cumulants [10-13], wavelets [14-18], spectral peaks [19], etc. The classifier block makes use of extracted features to identify signal modulation by applying a fixed threshold, or alternatively using a pattern recognition technique, such as artificial neural networks [20-22] or support vector machines [18]. If the feature set is carefully selected, FB methods are more robust and offer low processing complexity. For more details about AMC methods with a comprehensive literature review, the reader is referred to [5,23].

Attempts to classify signals in HF noise have been reported in the literature. In [24-26], entropic distance feature has been exploited for a classification of constant envelope digital signals, such as PSK and FSK modulations. In [26], this feature has been further explored for signals propagating via multiple ionospheric modes with co-channel interference and non-Gaussian noise for different types of PSK and FSK modulations. The basic idea of entropic distance is to compress the received signal using a compression algorithm and use the compression ratio as entropy measure of the received signal of an unknown modulation type. Therefore, normalized entropic distance can be used to classify different modulation schemes. It is also demonstrated in $[24,26]$ that entropic distance is a useful feature in separating narrow band as well as wide band FSK modulations. Its robustness against parameters variations such as quantization resolution, signal length, and compression algorithm is also verified.

Motivated by the observations noted in [4], effect of BK distribution on AMC design has been investigated in $[27,28]$. The results in [27] show that the performance of an AMC algorithm designed for $\mathrm{G}$ noise model significantly deteriorates in presence of $\mathrm{BK}$ noise, specifically at low signal-to-noise ratio (SNR). In addition, the investigation in [28] shows that it is possible to design features that are reasonably robust in presence of HF noise.

The objective in this article is to develop a new decision tree-based AMC algorithm well-suited for the classification of most popular single carrier modulations used in HF communication systems, i.e., 2FSK, 4FSK, 8FSK, 2PSK, 4PSK, 8PSK, 16QAM, 32QAM, 64QAM, 16 amplitude-phase shift keying (APSK), and 32APSK [1]. APSK is a digital modulation scheme that can be considered as a class of QAM in which symbols are always placed on equidistant concentric circles in the constellation diagram. This modulation scheme is known to have fewer problems with nonlinear amplifiers due to its constellation shape [29].

To achieve the above objective, the following features are considered: the maximum value of power spectral density (PSD) of normalized-centered instantaneous amplitude, the maximum value of magnitude of discrete fourier transform (DFT) of $k$ th power of received signal $\left(\Gamma_{k}\right)$, and number of points in pre-defined ranges of partitioned signal constellation magnitude. The first feature is well known in the literature [7-9]. The second feature

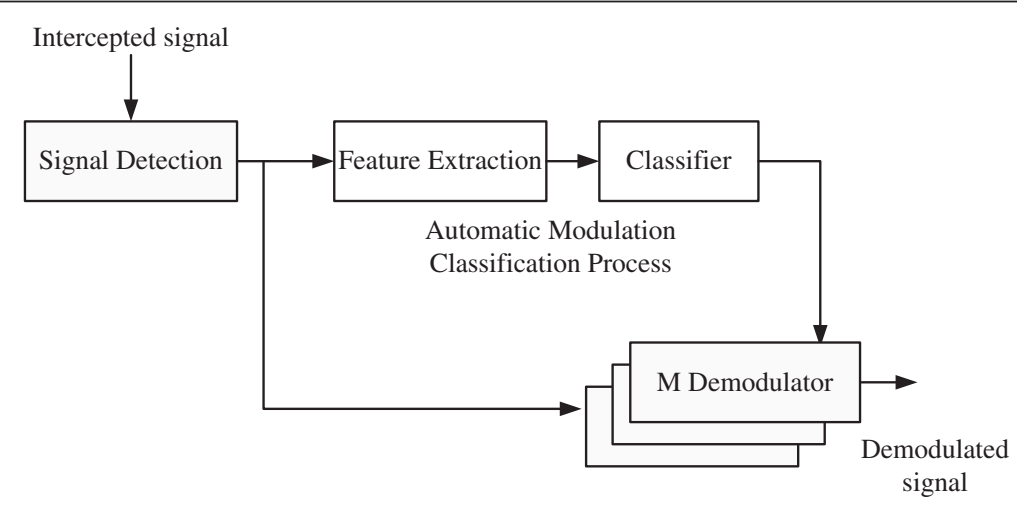

Figure 1 AMC-based receiver architecture using FB methods. 
with $k=2,4$ has previously been proposed in [30] for blind estimation of frequency offset of PSK and QAM signals and in [31] for the classification of MPSK modulations. The third feature is new; it is based on counting the number of points of the magnitude of received signal constellation in a certain predefined range. This feature has a desirable property in that its value remains almost constant even when the noise model gets changed from Gaussian distribution to BK distribution. Another important property of this feature is its low computational cost, as it only requires addition and comparison operations. It is worth noting that all three features are insensitive to symbol synchronization and carrier frequency and phase offsets.

The rest of the article is organized as follows. A unified mathematical model for all the modulation schemes under consideration is presented in Section 2. The proposed AMC and its computational complexity are detailed out in Section 3. Simulation results are presented and discussed in Section 4. Finally, conclusions are drawn in Section 5.

\section{Signal model}

The general form of received signal encompassing all modulation schemes under consideration is given by [32]

$$
r(t)=\operatorname{Re}\left\{\alpha e^{j 2 \pi \phi} e^{j 2 \Delta f t} C(t) e^{j 2 \pi f c\left(t-t_{0}\right)}\right\}+n(t)
$$

where $C(t)$ is the complex envelope of the modulated signal, $n(t)$ is a band-limited noise, $f_{\mathrm{c}}$ is the carrier frequency, $\alpha$ is the channel amplitude, $\phi$ is the phase offset, $\Delta f$ is the carrier frequency offset, and $\operatorname{Re}\{$.$\} denotes the$ real part. The complex envelope is characterized by the constellation points $C_{i}$, signal power $P$, and pulse shaping function $p(t)$. For $N$ symbols with periodicity $T$, the general form of the complex envelope can be expressed as

$$
C(t)=\sum_{i=1}^{N} C_{i} p(t-i T)
$$

In practice, $p(t)$ is often root-raised cosine with roll-off factor $(\rho)$ ranging between 0.2 and 0.5 [33-36]. For FSK modulation, rectangular pulse shape is commonly used [1]. The constellation points of digital modulation of order $M$ considered in this article are given in Table 1 (in Table $1, f_{\mathrm{s}}$ is the sampling frequency and $N_{\mathrm{f}}$ is the number of samples within one MFSK symbol duration); see also [9].

The noise term is often constructed by passing the BK/G noise through a band-limiting filter [9]. The bandwidth of this filter is set to $3 \mathrm{kHz}$ for HF channel [37]. This filter is used in practice to minimize the transmission bandwidth. The SNR at the output of band-limiting filter is adjusted to the desired value by multiplying the noise term by a scaling factor, $\beta$. That is [9]

$$
\beta=\sqrt{\frac{P}{N_{0}} 10^{-S N R / 20}}
$$

where $N_{0}$ is the noise power and SNR is in $\mathrm{dB}$.

\section{The proposed AMC}

\section{The proposed AMC features}

The proposed features and classification mechanism are described next. The presentation in this section is supported by simulation results conducted with parameters as described in Table 2, and averaged over 100 independent realizations. The frequency offset is set at 100 $\mathrm{Hz}$ and roll-off factor $\rho=0.35$. If different, results are presented for both $\mathrm{G}$ and $\mathrm{BK}$ noise models, otherwise results of BK noise model are only displayed.

\section{The maximum value of PSD of normalized-centered instantaneous amplitude}

This feature is expressed mathematically as [9]

$$
\gamma_{\text {max }}=\frac{\max \left|D F T\left(a_{c n}(n)\right)\right|^{2}}{N_{s}}
$$

where $N_{\mathrm{s}}$ is the number of samples, $a_{c n}(n)=a(n) / m_{a}-$ $1, a(n)$ is the absolute value of the analytic form of the received signal, and $m_{a}$ is its sample mean. This feature classifies PSK modulations as one group, and FSK and OQPSK modulations as another group. Figure 2 shows feature values against SNR (in dB) for PSK, FSK, and OQPSK signals. The figure also shows the mean value of feature (solid line) \pm its standard deviation (dotted line). This will help in determining the bounds for feature variation. Note that we only display bounds (mean \pm its

Table 1 Constellation points of digital modulation schemes

\begin{tabular}{ll}
\hline Modulation & Constellation point \\
\hline MPSK & $C_{i} \in \exp (-j 2 \pi m / M), m=0,1, M-1$ \\
MQAM & $C_{i}=a_{K}+j b_{k}, a_{k}, b_{k} \in\{2 m-1-\sqrt{M}\}, \quad m=1,2, ., \sqrt{M}$ \\
OQPSK & $C_{i} \in 4$ PSK staggered to allow $\pm \pi / 2$ change between symbols. \\
MFSK & $C_{i} \in \cos \left(\frac{2 \pi f_{m}}{f_{s}} n\right)+j \sin \left(\frac{2 \pi f_{m}}{f_{s}}\right) ; \cdot m \in\{0, \ldots, M-1\}, n=0,1, \ldots, N_{f}-1$ \\
MAPSK & $C_{i} \in \exp \left(-\frac{j 2 \pi m}{M_{s}}\right)$, where $\left\{\begin{array}{l}r \in[1,2], \quad M_{s} \in[4,12], \quad m \in[0, \ldots, 11], \text { if } M=16 \\
r \in[1,2,3], \quad M_{s} \in[4,12,16], \quad m \in[0, \ldots, 15], \text { if } M=32\end{array}\right\}$ \\
\hline
\end{tabular}


Table 2 Simulation parameters

\begin{tabular}{ll}
\hline Parameter & Value \\
\hline Carrier frequency & $f_{\mathrm{c}}=24 \mathrm{kHZ}$ \\
Symbol rate & $r_{\mathrm{s}}=2400 \mathrm{~Hz}$ \\
Sampling rate & $f_{\mathrm{s}}=19.2 \mathrm{kHz}$ \\
Number of symbols & $\mathrm{N}=512$ \\
Total number of samples & $N_{\mathrm{s}}=4096$ \\
\hline
\end{tabular}

standard deviation) for the modulations that are critical for determining the thresholds. For the sake of clarity, the same color has been used for both the bound and the mean that belong to a particular modulation. As Figure 2 shows, the final classification of the modulations is achieved using a pre-computed threshold which separates PSK from FSK and OQPSK modulations.

The maximum value of DFT magnitude of the kth power of analytic form of received signal

This feature is defined as follows [30,31].

$$
\Gamma_{k}=\frac{\max \left|\operatorname{DFT}\left(a(i)^{k}\right)\right|^{2}}{N_{s}}
$$

Three values of parameter $k$ are used in this article, i.e., $k=1,2$, and 4 . The three FSK modulation orders are classified using $\Gamma_{1}$. Since the input signal power is equally distributed among all FSK tones, therefore for a certain fixed duration of the received signal the feature value is highest for 2FSK and lowest for 8FSK and OQPSK. As a result, 2FSK, 4FSK, and group of 8FSK and OQPSK can be discriminated using two threshold values. The values of this feature against SNR are shown in Figure 3. Also, the upper and lower bounds for both thresholds are shown as dotted lines.
The feature $\Gamma_{k}$ with $k=2$ and 4 is utilized to classify the three individual PSK modulations in two stages. In the first stage, 2PSK is separated from 4PSK and 8PSK by using $\Gamma_{2}$, whereas in the second stage 4PSK is separated from 8PSK modulation by using $\Gamma_{4}$. Both $\Gamma_{2}$ and $\Gamma_{4}$ are compared against two different thresholds. Figures 4 and 5 show the variation of $\Gamma_{2}$ and $\Gamma_{4}$ against SNR, respectively. It is observed that these features have the ability to separate PSK modulations at SNR as low as 0 $\mathrm{dB}$. It is also used to classify OQPSK and 8FSK modulations. Figure 6 shows that $\Gamma_{4}$ can classify both modulations at $\mathrm{SNR}=0 \mathrm{~dB}$.

Finally, feature $\Gamma_{4}$ can be further used to separate 16/ 64QAM from 16/32APSK and 32QAM modulations. The threshold for separating the two groups is set to allow classification starting from around $0 \mathrm{~dB}$ by carefully taking into consideration the variance of feature values. Note that the variance of $\Gamma_{4}$ for $64 \mathrm{QAM}$ is larger than that of 32QAM as shown in Figure 7.

\section{Number of points of partitioned magnitude of constellation diagram}

QAM/APSK modulation symbols are defined in terms of phase and amplitude variations and are represented in the form of a constellation diagram. This diagram is extracted from the analytic form of the IF signal after down conversion to the baseband. Many features exist in literature that exploit different aspects of the constellation diagram for classification of 16, 32, and 64QAM modulations [38-42]. In this article, a new feature based on partitioning magnitude of constellation diagram is proposed. This feature makes use of the observation that the noise-free normalized constellation points of PSK and FSK modulations are on the unit circle, whereas the normalized constellation points of QAM and APSK

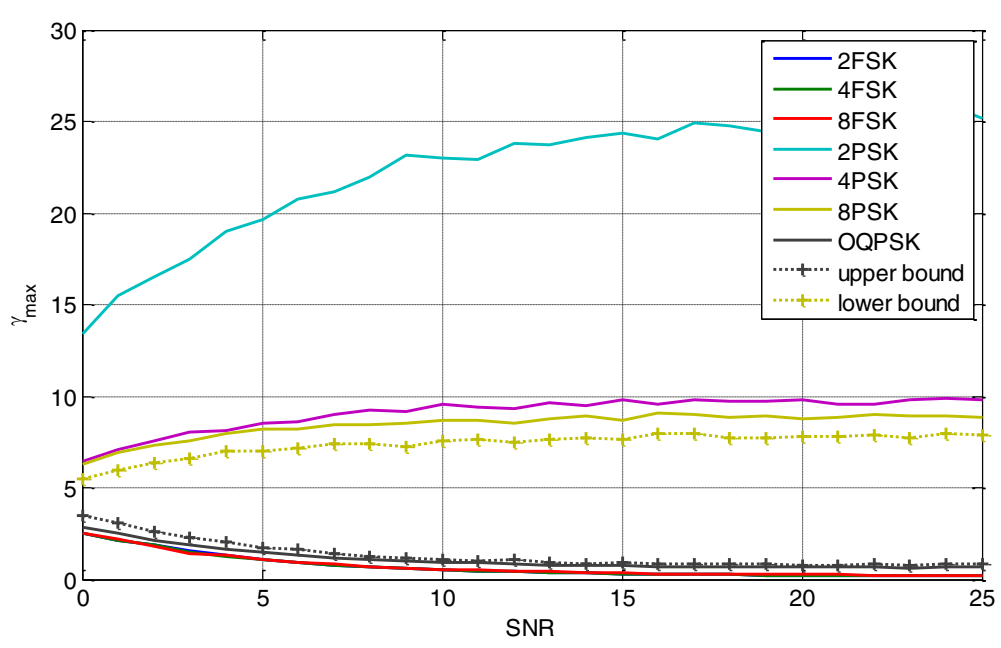

Figure $2 \gamma_{\max }$ versus SNR for FSK, PSK, and OQPSK signals. 


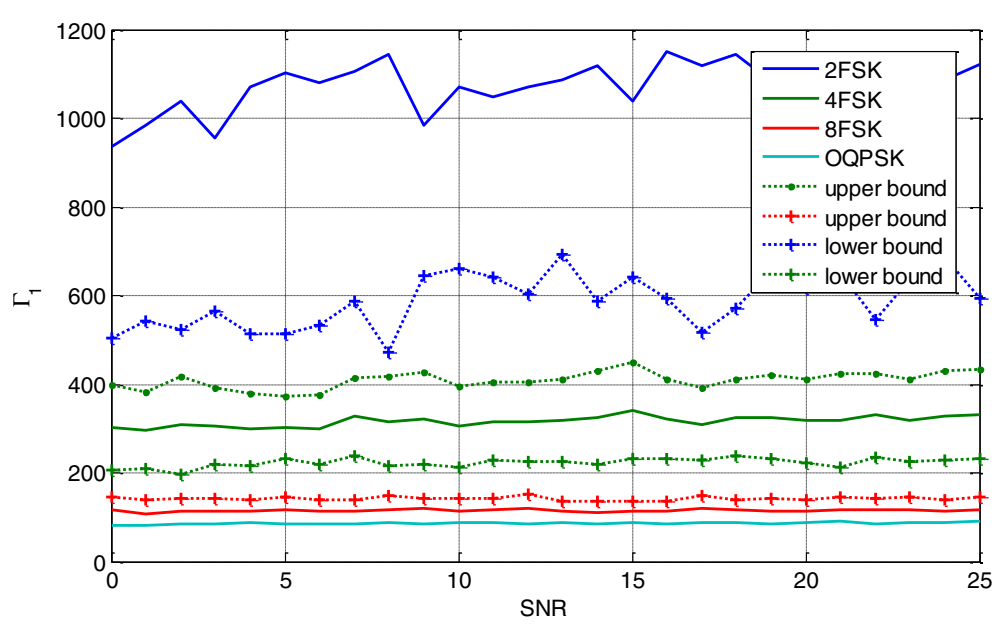

Figure $3 \Gamma_{1}$ versus SNR for the three FSK signals.

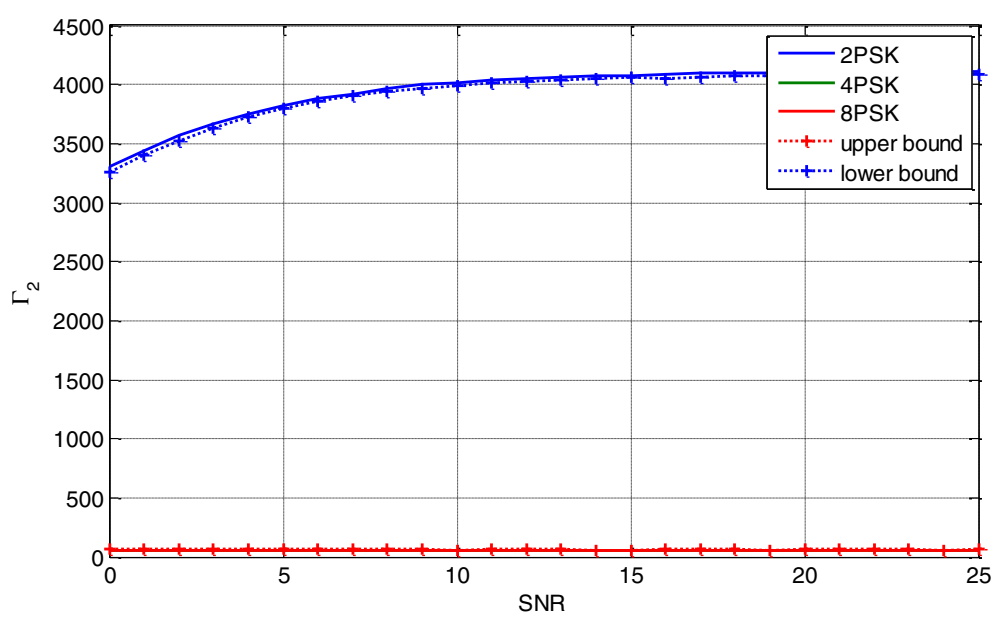

Figure $4 \Gamma_{2}$ versus SNR for the three PSK signals.

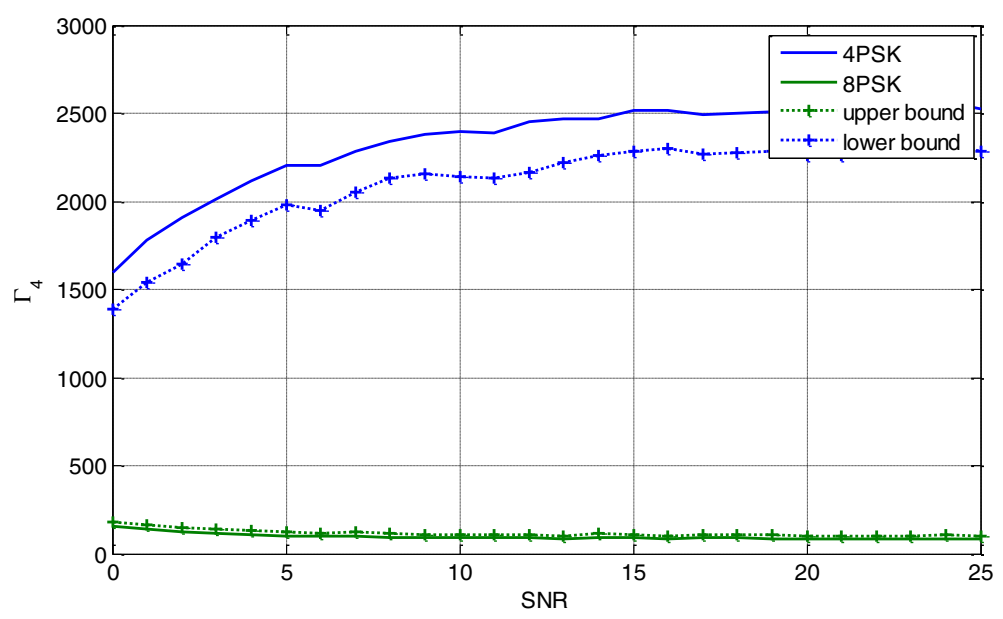

Figure $5 \Gamma_{4}$ versus SNR for 4PSK and 8PSK signals. 


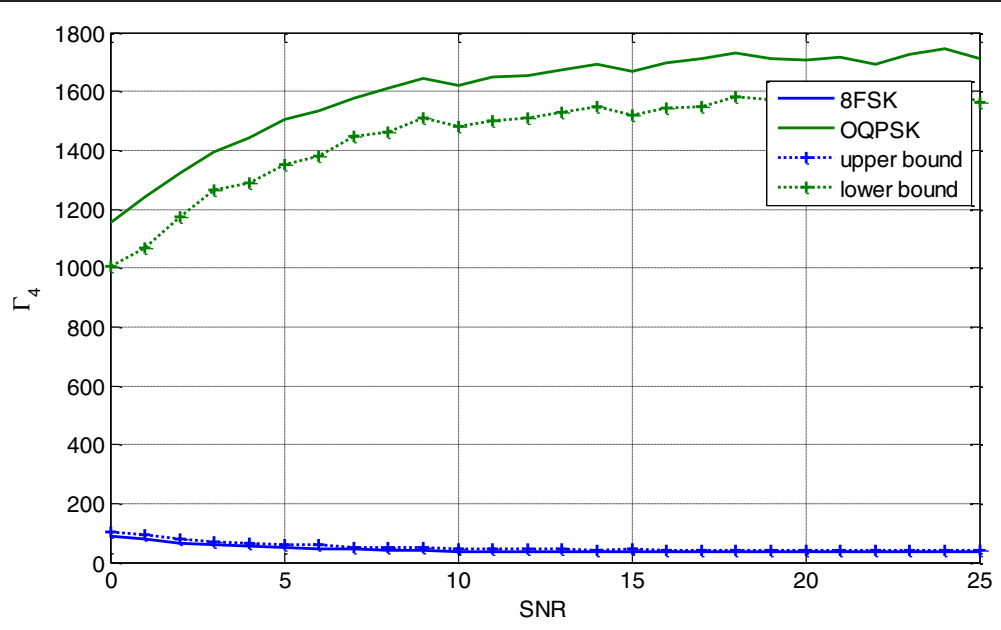

Figure $6 \Gamma_{4}$ versus SNR for 8FSK and OQPSK signals.

modulations may lie on, inside, and/or outside the unit circle. Therefore, this feature is useful in separating near constant amplitude modulations from amplitude varying modulations, and also in determining the order of amplitude varying modulations.

The first step of proposed feature is to partition the magnitude of normalized constellation diagram into seven regions denoted by $R_{1}, R_{2}, \ldots, R_{7}$. Note that the samples of QAM/APSK signal are normalized by the standard deviation of the signal amplitude before feature extraction. Then, the signal is classified by comparing the number of points in one or more pre-defined regions to a pre-computed threshold. Table 3 shows the boundaries of these regions which are determined by examining the histogram of magnitude of normalized constellation diagram of QAM/APSK modulations. To ensure maximum separation between different modulations, their respective histograms are searched for regions in which the number of points differs by large values across different histograms.

Let $N_{i}(i=1,2, \ldots, 7)$ denote the normalized number of constellation points whose magnitude falls within the range $R_{i}$. The normalization pertaining to $N_{i}$ is performed with respect to the total number of received signal samples $N_{\mathrm{s}}$. Therefore, we can define the following features.

$$
\begin{aligned}
& K_{1}=N_{4}+N_{5} \\
& K_{2}=N_{1}+N_{2}+N_{5} \\
& K_{3}=N_{6} \\
& K_{4}=N_{2}+N_{7}
\end{aligned}
$$

Figure 8 shows the variation of feature $K_{1}$ extracted from PSK, FSK, QAM, and APSK signals at different SNR values. The threshold is selected to separate QAM/ APSK from rest of the modulation schemes. It can be

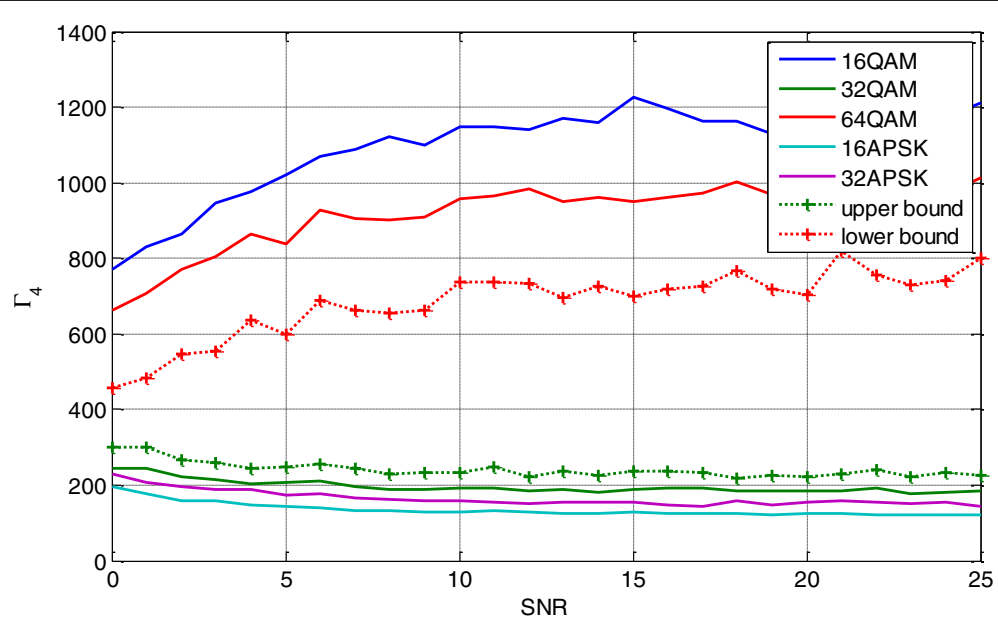

Figure $7 \Gamma_{4}$ versus SNR for QAM and APSK signals. 
Table 3 Regions of partitioned magnitude of constellation diagram

\begin{tabular}{lll}
\hline Region & Range & \\
\cline { 2 - 3 } Starting point & Ending point \\
\hline$R_{1}$ & 0 & 0.2 \\
$R_{2}$ & 0.2 & 0.3 \\
$R_{3}$ & 0.3 & 0.4 \\
$R_{4}$ & 0.4 & 0.6 \\
$R_{5}$ & 0.6 & 0.8 \\
$R_{6}$ & 1.00 & 1.25 \\
$R_{7}$ & 1.6 & 1.7 \\
\hline
\end{tabular}

observed from Figure 8 that with this feature, successful classification can be obtained at SNR greater than $4 \mathrm{~dB}$. By virtue of Figure 8, it is also observed that noise statistics dominate the feature $K_{1}$ at values of SNR less than 4 $\mathrm{dB}$, which makes it impossible to distinguish at this range one modulation type from another.

Figure 9 shows the normalized histogram of magnitude of constellation diagram of 16QAM and 64QAM signals computed in the absence of noise. Based on this figure, it is obvious that 16QAM is separable from 64QAM by making use of the feature $K_{2}$. Figure 10 shows the variation of the feature $K_{2}$ against SNR, where it is observed that successful classification can be

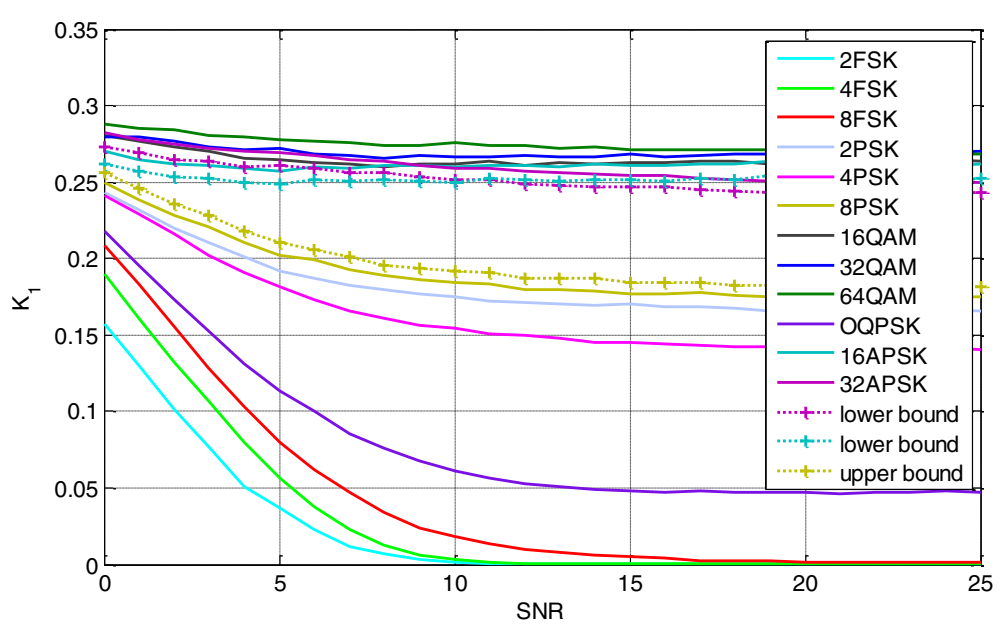

Figure $8 K_{1}$ versus SNR for the different FSK/PSK and QAM/APSK signals.

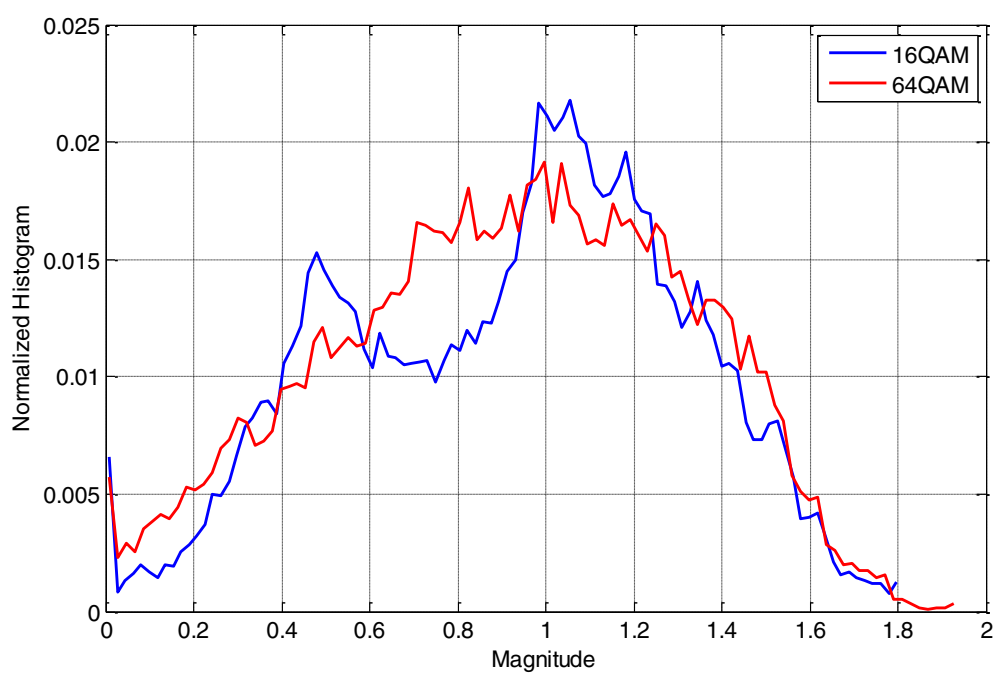

Figure 9 Normalized histograms for constellation points of noiseless 16QAM and 64QAM signals. 


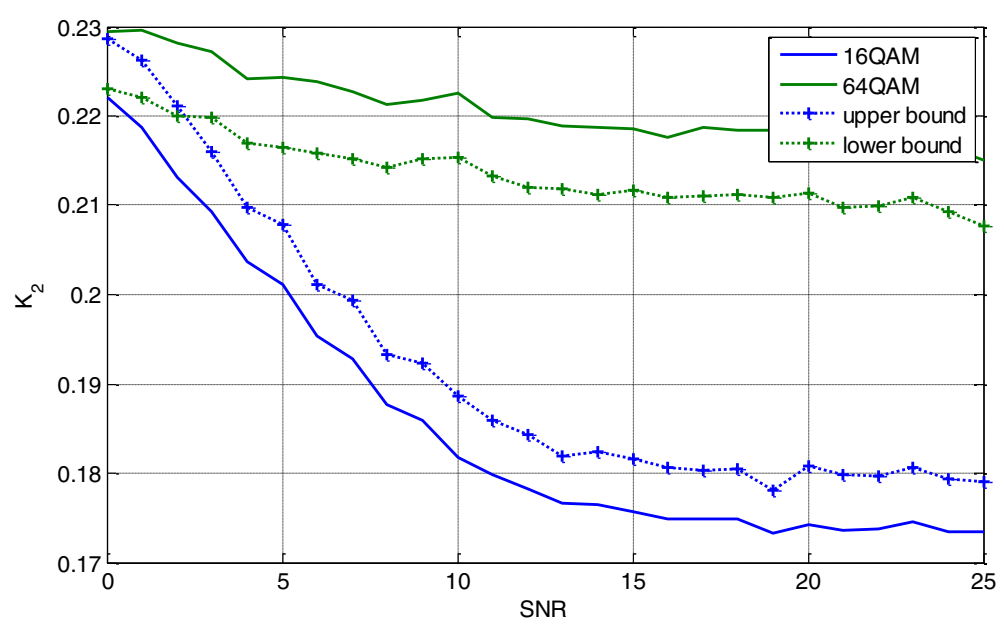

Figure $10 K_{2}$ versus SNR for 16QAM and 64QAM signals.

achieved at approximately $7 \mathrm{~dB}$. 16APSK is separable from 32QAM/APSK using feature $K_{3}$ which is identified by examining the normalized histogram of the constellations of respective modulations displayed in Figure 11. Figure 12 shows the variation of the feature $K_{3}$ against SNR, which shows good prospects of classification around $3 \mathrm{~dB}$. Finally, 32APSK and 32QAM are separated using $K_{4}$. Note that $K_{4}$ is effective at approximately $\mathrm{SNR}=13 \mathrm{~dB}$ as shown in Figure 13.

\section{Structure of the AMC algorithm}

The proposed AMC follows a step-by-step approach for classification of the modulation schemes. A decision is taken at each step using one of the features which results in identification of either a type or order of an individual modulation. There are a total of three main features used to classify 12 modulation schemes targeted in this article. The structure of the decision tree is illustrated in Figure 14. Each decision node (comparator) is labeled with the name of the feature. It can be observed that in the initial stages only modulation types are identified, and orders of the individual modulation schemes are determined in later stages.

\section{Computational complexity}

The computational complexity of the proposed AMC algorithm determines its suitability for real-time implementation in practical systems. Here, we consider the algorithm complexity in terms of number of multiplications, additions, and comparisons given the sequence $a_{\mathrm{cn}}(i)$ or $a(i)$. Table 4 shows these counts expressed in terms of number of samples $N_{\mathrm{s}}$. It is of interest to note that the proposed feature has the least computational cost as it requires no multiplication operations. By virtue

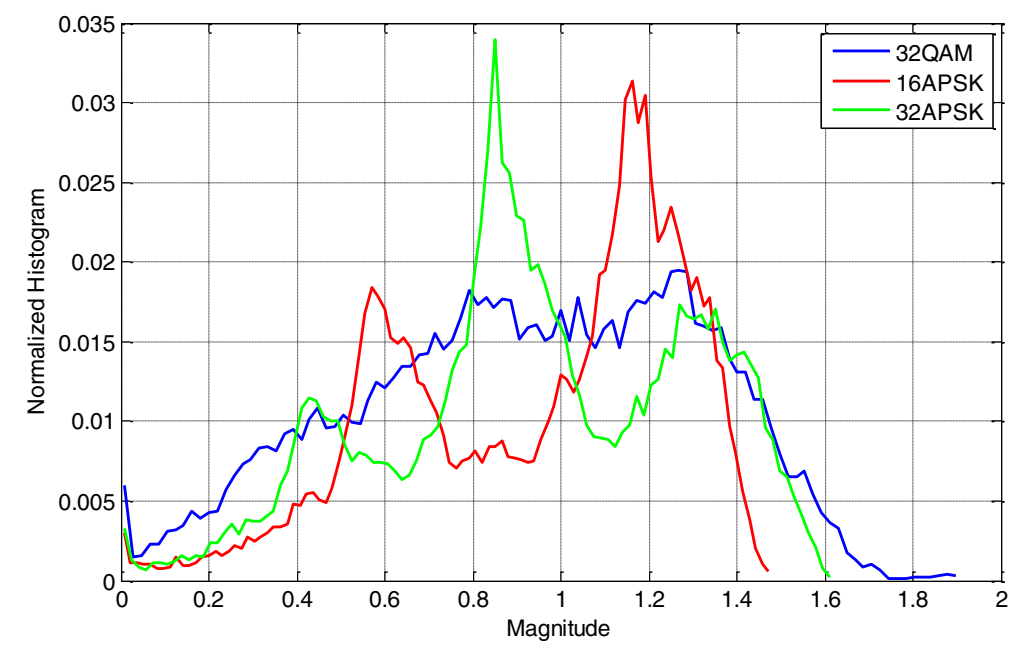

Figure 11 Normalized histograms for the magnitude of the constellation points of noiseless 32QAM, 16APSK, and 32APSK signals. 


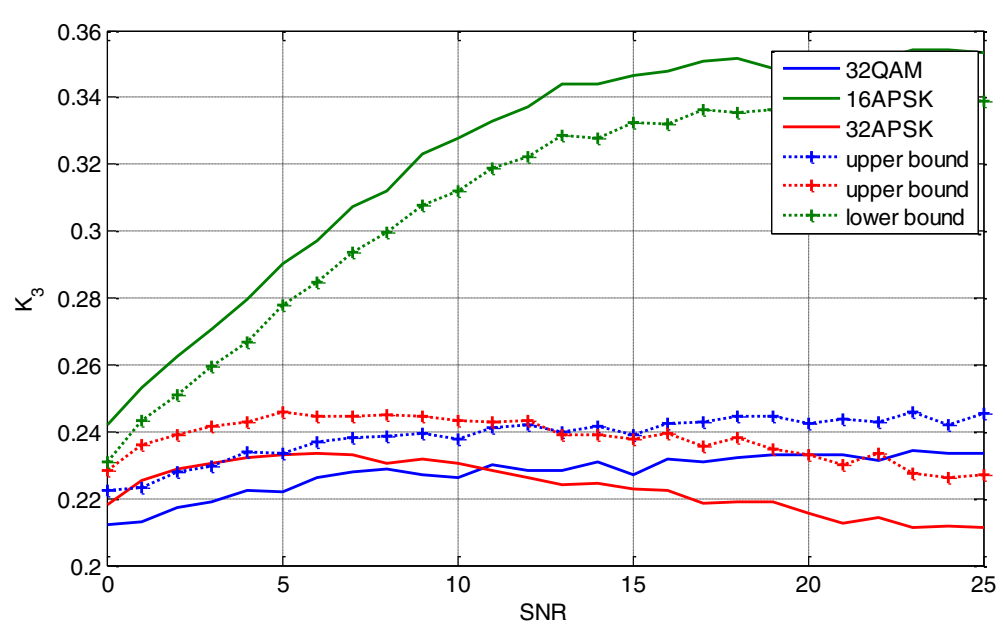

Figure 12 K3 versus SNR for 16APSK, 32APSK, and 32QAM signals.

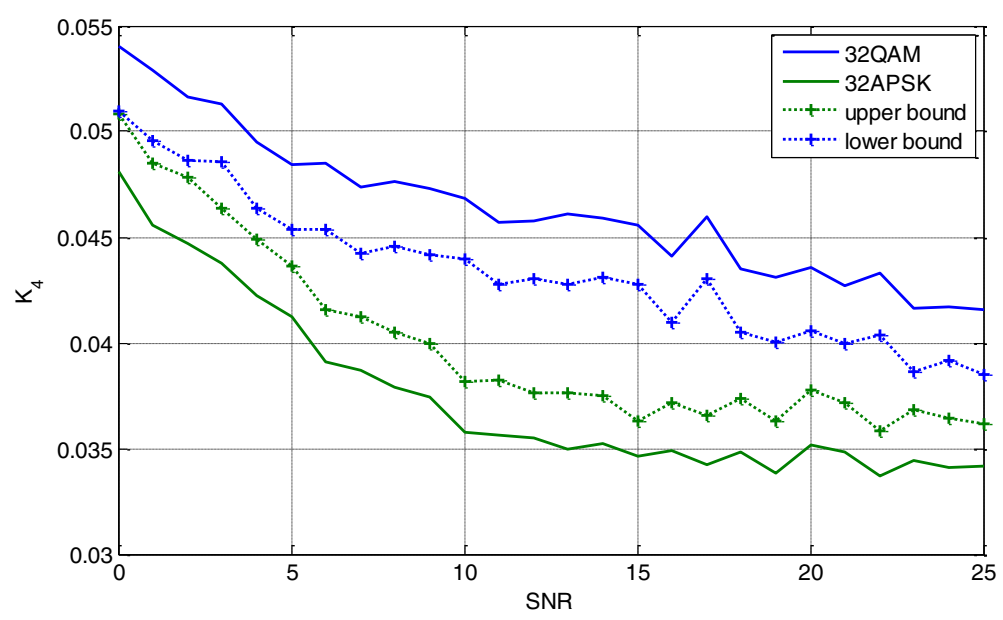

Figure $13 K_{4}$ versus SNR for 32APSK and 32QAM signals.

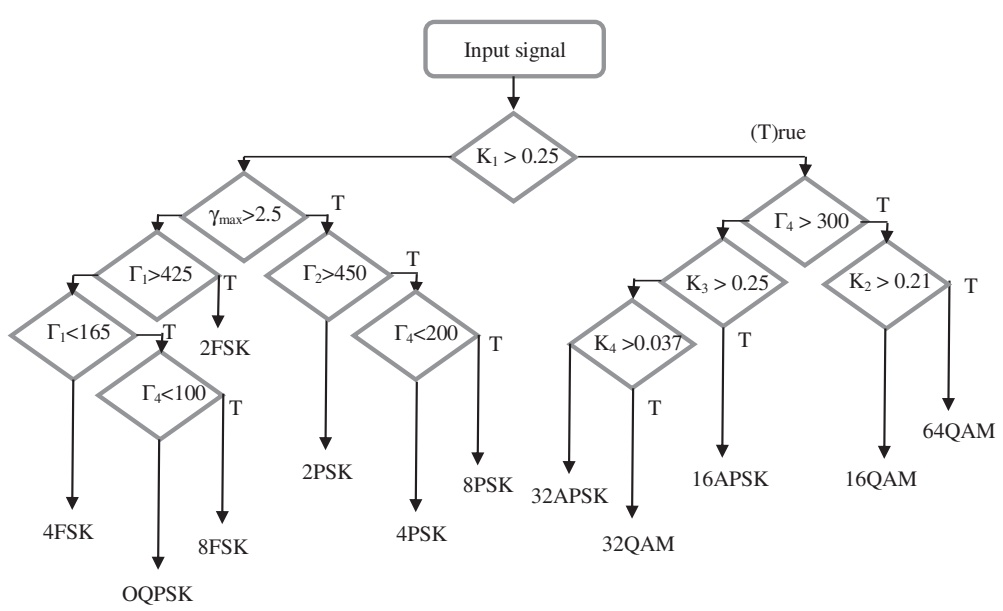

Figure 14 The flow chart of the proposed AMC algorithm. 
Table 4 Computational complexity of the proposed algorithm

\begin{tabular}{llll}
\hline Feature & \multicolumn{2}{l}{ Number of operations } & \\
\cline { 2 - 4 } \cline { 2 - 4 } & Complex multiplication & Complex addition & Comparison \\
\hline$K_{1}$ & 0 & $N_{\mathrm{s}}-1$ & $2 N_{\mathrm{s}}+1$ \\
$K_{2}$ & 0 & $N_{\mathrm{s}}-1$ & $3 N_{\mathrm{s}}+1$ \\
$K_{3}$ & 0 & $N_{\mathrm{s}}-1$ & $2 N_{\mathrm{s}}+1$ \\
$K_{4}$ & 0 & $N_{\mathrm{s}}-1$ & $4 N_{\mathrm{s}}+1$ \\
$K_{\max }$ & $N_{\mathrm{s}} / 2 \log \left(N_{\mathrm{s}}\right)+N_{\mathrm{s}}+1$ & $N_{\mathrm{s}} \log \left(N_{\mathrm{s}}\right)$ & $N_{\mathrm{s}}$ \\
$\Gamma_{1}$ & $N_{\mathrm{s}} / 2 \log \left(N_{\mathrm{s}}\right)+N_{\mathrm{s}}+1$ & $N_{\mathrm{s}} \log \left(N_{\mathrm{s}}\right)$ & $N_{\mathrm{s}}$ \\
$\Gamma_{2}$ & $N_{\mathrm{s}} / 2 \log \left(N_{\mathrm{s}}\right)+2 N_{\mathrm{s}}+1$ & $N_{\mathrm{s}} \log \left(N_{\mathrm{s}}\right)$ & $N_{\mathrm{s}}$ \\
$\Gamma_{4}$ & $N_{\mathrm{s}} / 2 \log \left(N_{\mathrm{s}}\right)+4 N_{\mathrm{s}}+1$ & $N_{\mathrm{s}} \log \left(N_{\mathrm{s}}\right)$ & $N_{\mathrm{s}}$ \\
\hline
\end{tabular}

of Table 4, it is not difficult to conclude that the computational complexity is upper bounded by $N_{\mathrm{s}} / 2 \log \left(N_{\mathrm{s}}\right)+$ $4 N_{\mathrm{s}}+1$ multiplications, $N_{\mathrm{s}} \log \left(N_{\mathrm{s}}\right)$ additions and $4 N_{s}+1$ comparisons, which is very attractive for real-time implementation. Note that Table 4 shows the maximum possible addition operations for the proposed feature $K_{l}$. In reality, far fewer additions are needed.

\section{Results}

This section presents results that show the overall performance of the proposed AMC algorithm in the presence of either $\mathrm{G}$ or BK noise for classifying the type and order of a particular modulation. Features described in Section 3 are extracted from the signal under consideration and utilized in the proposed AMC scheme according to Figure 14. Simulation results are presented at different values of SNR using parameters' values given in Table 2. The performance is measured in terms of probability of correct classification $\left(P_{\mathrm{cc}}\right)$ averaged over 100 independent trials.

Figures 15 and 16 show the classification rate of QAM/APSK signals, where it is observed that 16QAM and 32APSK have the lowest values. Specifically, 16QAM and 32APSK have $P_{\mathrm{cc}}>0.9$ at $\mathrm{SNR}=12 \mathrm{~dB}$ and $\mathrm{SNR}=13 \mathrm{~dB}$, respectively. On the other hand, 64QAM has shown almost constant performance with respect to SNR. This is intuitively not surprising as the steps pertaining to 64QAM classification first test the received signal against the hypothesis of being 32QAM/32APSK or 16APSK. If not true, then the signal is tested against the hypothesis of being 16QAM; otherwise it is declared 64QAM. That is, as the SNR decreases, the likelihood of correct classification is always in favor of 64QAM. Similar conclusion can be drawn for 32QAM.

Figure 17 shows the classification rate of FSK and OQPSK modulations in the presence of G/BK noise, where $P_{\mathrm{cc}}>0.9$ is attained for both noise models at approximately $\mathrm{SNR}=7 \mathrm{~dB}$. Similar performance has been observed for PSK with $P_{\mathrm{cc}}=1$ at $\mathrm{SNR}=8 \mathrm{~dB}$, as shown in Figure 18.

The average probability of correct classification $\left(P_{\text {ca }}\right)$ over all 12 modulation schemes is shown in Figures 19, 20, and 21 for different noise distributions, roll-off factor, and frequency offsets, respectively. It is observed that change of noise model or frequency offset has almost no significant effect on the classification rate. In addition, the proposed AMC method shows acceptable performance with practical values of roll-off factor.

Figure 22 shows $P_{\mathrm{ca}}$ of the proposed AMC algorithm at two different symbol rates: 1200 and $2400 \mathrm{~Hz}$ using the same thresholds computed at $2400 \mathrm{~Hz}$. The result shows slight discrepancies between the performances

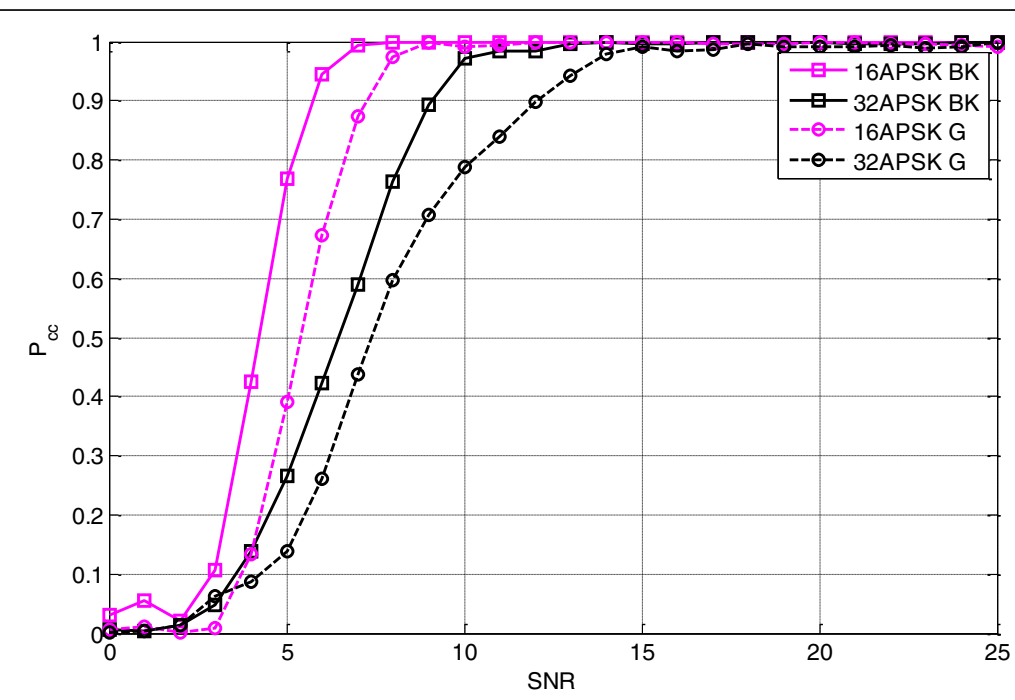

Figure 15 Performance of the proposed AMC algorithm pertaining to the classification of APSK modulations in presence of G and BK noises. 


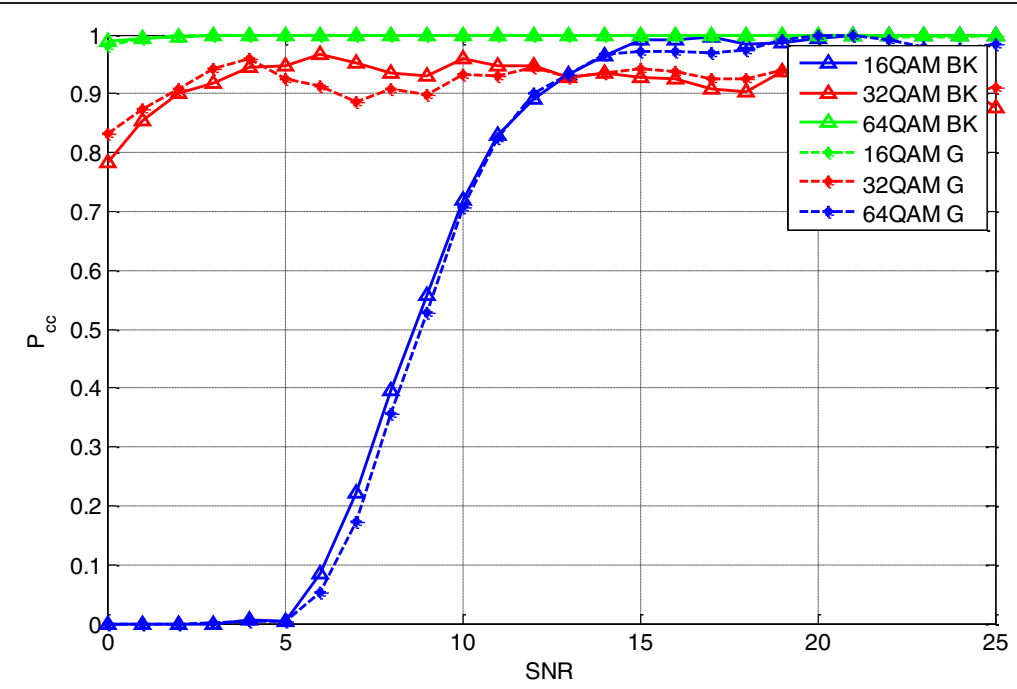

Figure 16 Performance of the proposed AMC algorithm pertaining to the classification of QAM modulations in presence of G and BK noises.

using the two data rates. Note that as the data rate decreases, the number of symbols involved in features' computations also decreases for fixed values of $N_{\mathrm{s}}$ and $f_{\mathrm{s}}$. Changing the sampling rate also affects the algorithm in a similar manner to the effect of changing the symbol rate. In fact, the main influencing factor is the number of samples per symbol. The proposed AMC algorithm makes use of a fixed window of size $N_{\mathrm{s}}$ received samples. Increasing (deceasing) the sampling rate means fewer (more) symbols are packed into the $N_{\mathrm{s}}$ samples window. Therefore, it can be inferred from Figure 22 the effect of changing the sampling rate for fixed values of $N_{\mathrm{s}}$. By virtue of Figure 22, it is evident that for optimum results, thresholds need to be fine-tuned if the sampling rate gets changed.

\section{Conclusions}

In this article, a new features-based decision tree AMC algorithm is developed for the classification of most popular single carrier modulations used in HF communications systems, i.e., 2FSK, 4FSK, 8FSK, 2PSK, 4PSK, 8PSK, 16QAM, 32QAM, 64QAM, 16APSK, and 32APSK. Towards this objective, three features are employed that include PSD and DFT of $k$ th power of received signal. A new constellation-based feature for the classification of QAM and APSK signals is also proposed.

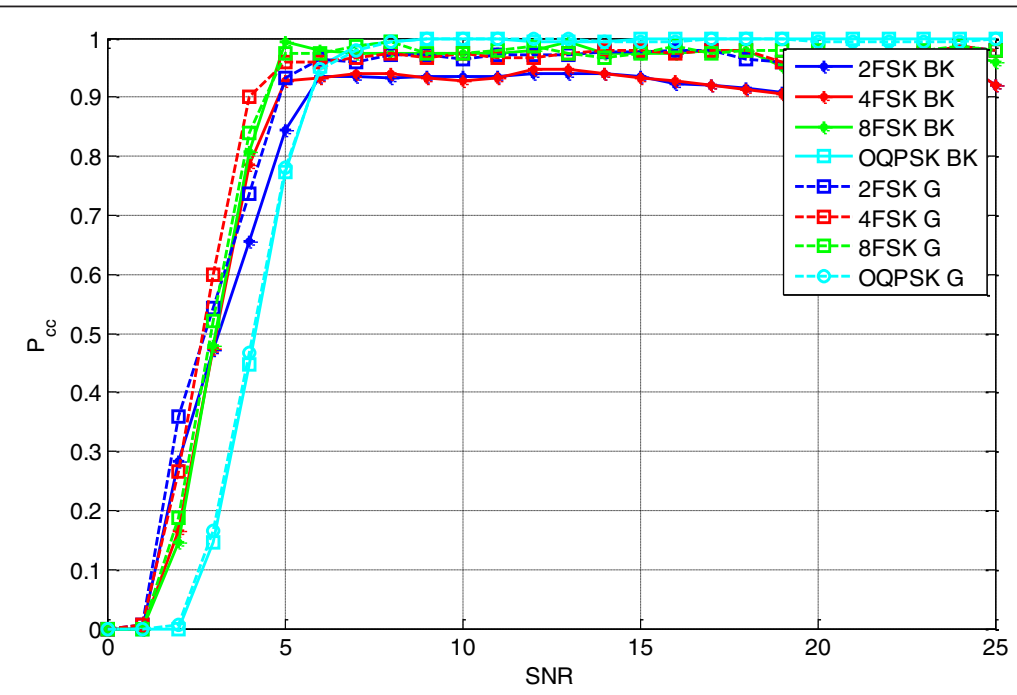

Figure 17 Performance of the proposed AMC algorithm pertaining to the classification of FSK and OQPSK modulations in presence of $G$ and BK noises. 


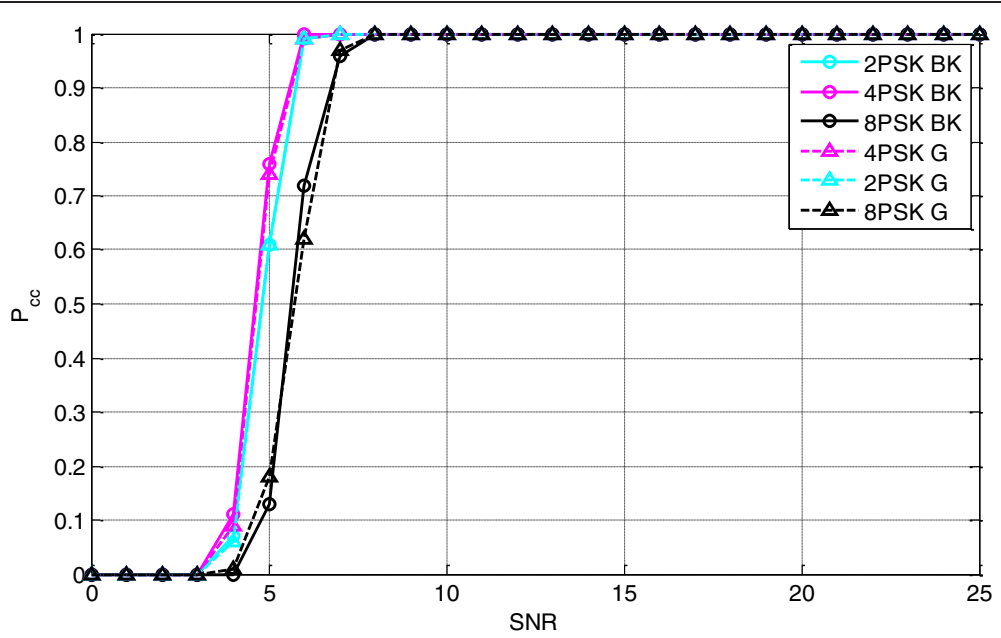

Figure 18 Performance of the proposed AMC algorithm pertaining to the classification of PSK modulation in presence of G and BK noises.

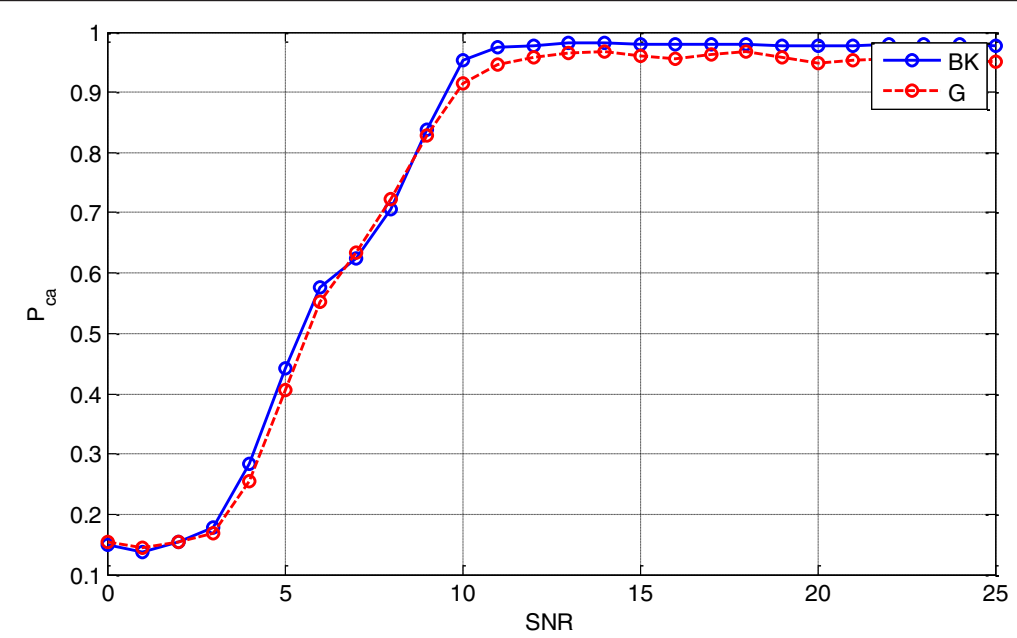

Figure 19 Average $P_{\mathrm{cc}}$ in presence of $\mathrm{G}$ and $\mathrm{BK}$ noises.

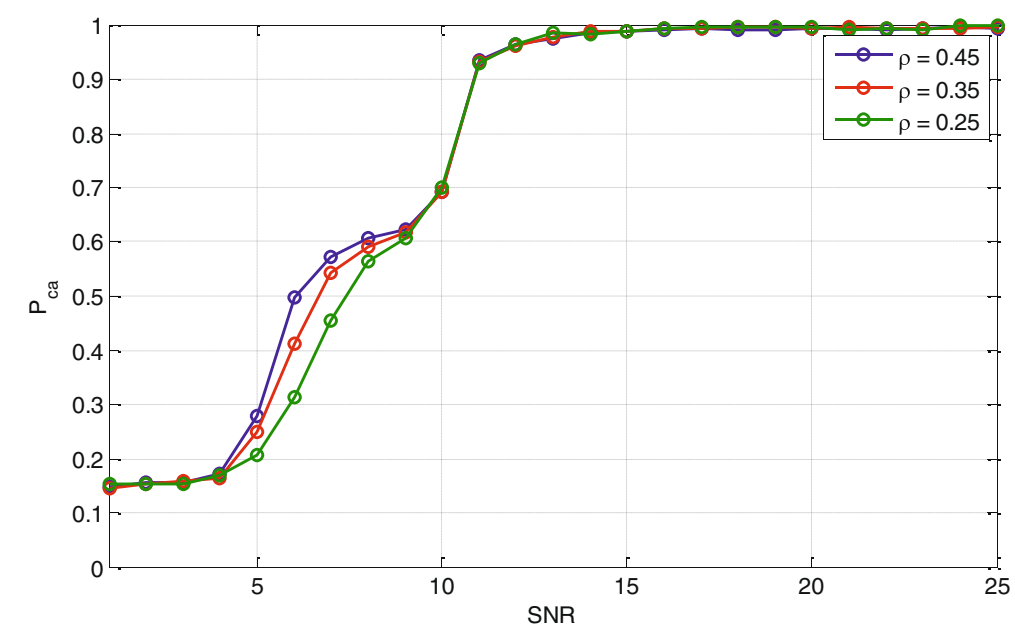

Figure 20 Average $P_{c c}$ with values of roll-off factor $0.25,0.35$, and 0.45 . 


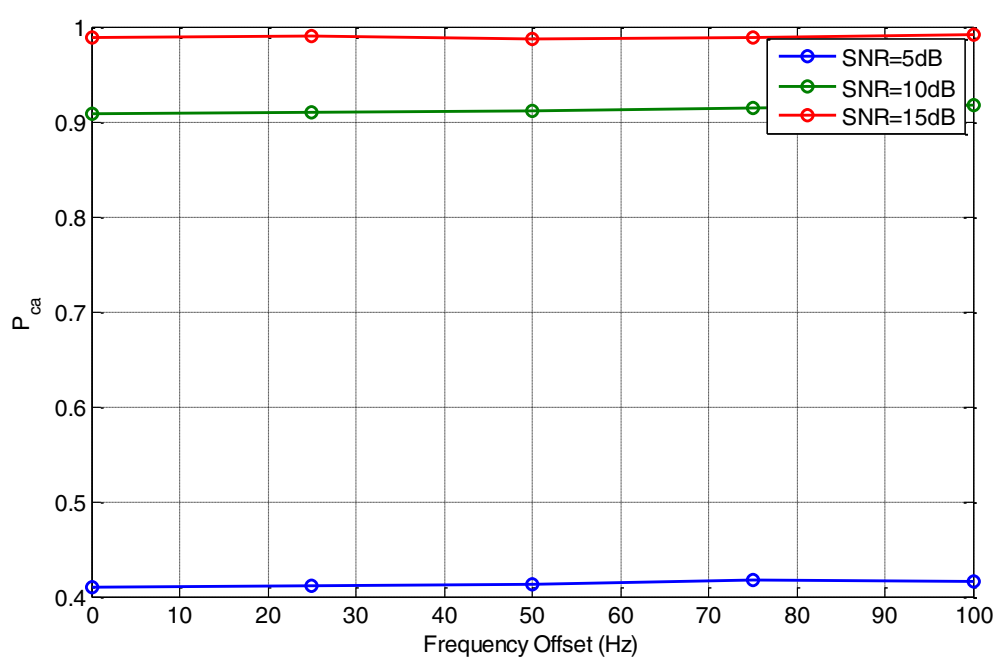

Figure 21 Average $P_{\mathrm{cc}}$ for different values of frequency offset.

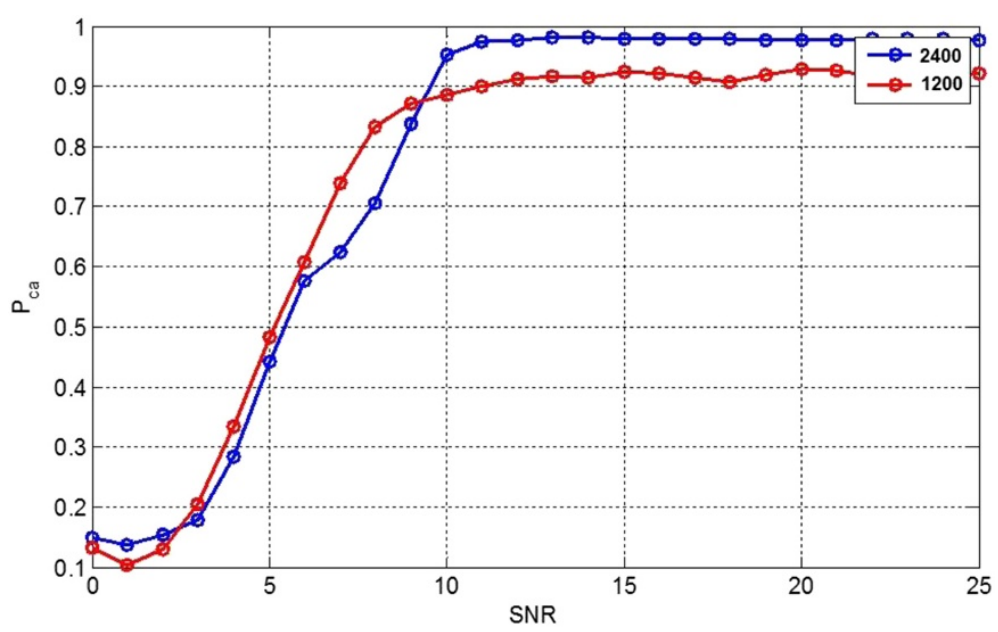

Figure 22 Average $P_{\mathrm{cc}}$ for different symbol rate values.

In practice, the received signal is often corrupted by HF noise whose statistical characteristics have PDF best described by a model that fluctuates between G and BK distributions; in addition the received signal may have frequency, phase, and symbol synchronization errors. Extensive simulations results have shown that the proposed features are insensitive to noise model variation or synchronization errors.

The proposed AMC method has an advantage of being simple to implement as it uses decision-tree with pre-computed thresholds for signal classification. In addition, it is capable to classify type and order of modulation in band-limited HF noise environment at relatively low SNR.

\section{Competing interests}

The authors declare that they have no competing interests.

\section{Acknowledgments}

The authors would like to thank the reviewers for their time and effort spent in carefully reviewing the manuscript, and for their valuable comments that have greatly contributed to the enhancement of article's quality. This study was supported by Grant no. 08-ELE 263-2 from the unit of Science and Technology at King Saud University.

\section{Author details}

${ }^{1}$ KACST Technology Innovation Center in Radio Frequency and Photonics, Riyadh, Saudi Arabia. ${ }^{2}$ Prince Sultan Advanced Technologies Research Institute/STC-Chair, Riyadh, Saudi Arabia. ${ }^{3}$ Electrical Engineering Department, King Saud University, Riyadh, Saudi Arabia.

Received: 13 March 2012 Accepted: 18 October 2012

Published: 14 November 2012

\section{References}

1. R. Prosch, Technical Handbook for Radio Monitoring I (Books on Demand $\mathrm{GmbH}$, Norderstedt, Germany, 2007)

2. N. Maslim, HF Communications: A Systems Approach (CRC Press, London, 1987) 
3. J. Giesbrecht, R. Clarke, D. Abbott, An empirical study of the probability density function of HF noise. Fluct. Noise Lett. 6(2), L117-L123 (2006)

4. J. Giesbrecht, An impirical study of HF noise near Adelaide Australia. Proc. IET International Conference on lonospheric Radio Systems and Techniques (IRST'09) (, Edinburgh, UK, 2009), pp. 310-314

5. J.L. Xu, W. Su, M. Zhou, Likelihood-ratio approaches to automatic modulation classification. IEEE Trans. Syst. Man Cybern. C Appl. Rev. 1, 455-469 (2011)

6. P. Jain, R.M. Buehrer, Implementation of adaptive modulation on the Sunrise software radio. Proc. 45th Midwest Symp. Circ. and Syst. (MWSCAS) 3, 405-408 (2002)

7. L. De Vito, S. Rapuano, M. Villanacci, Prototype of an automatic digital modulation classifier embedded in a real-time spectrum analyzer. IEEE Trans. Instrum. Meas. 59(10), 2639-2651 (2010)

8. A.K. Nandi, E.E. Azzouz, Algorithms for automatic modulation recognition of communication signals. IEEE Trans. Commun. 46, 431-436 (1998)

9. E.E. Azzouz, A.K. Nandi, Automatic Modulation Recognition of Communication signals (Kluver Academic, 1996)

10. V.D. Orlic, M.L. Dukic, Multipath channel estimation algorithm for automatic modulation classification using sixth-order cumulants. IET Electron. Lett. 46(19), 1348-1349 (2010)

11. H.-C. Wu, M. Saquib, Y. Zhifeng, Novel automatic modulation classification using cumulant features for communications via multipath channels. IEEE Trans. Wirel. Commun. 7(8), 3098-3105 (2008)

12. O.A. Dobre, A. Abdi, Y. Bar-Ness, W. Su, Selection combining for modulation recognition in fading channels. Proc. IEEE Military Communs. Conf. (MILCOM'05), vol. 4 (, Atlantic City, US, 2005), pp. 2499-2505

13. Z. Xin, W. Ying, Y. Bin, Signal classification method based on support vector machine and high-order cumulants. Wirel. Sensor Netw. 2(1), 48-52 (2010)

14. E. Avci, D. Hanbay, A. Varol, An expert discrete wavelet adaptive network based fuzzy inference system for digital modulation recognition. Expert Syst. Appl. 33(3), 582-589 (2007)

15. E. Avci, D. Avci, The performance comparison of discrete wavelet neural network and discrete wavelet adaptive network based fuzzy inference system for digital modulation recognition. Expert Syst. Appl. 35(1-2), 90-101 (2008)

16. K. Maliatsos, S. Vassaki, Ph. Constantinou, Interclass and Intraclass modulation recognition using the Wavelet Transformation. Proc. IEEE Personal, Indoor and Mobile Radio Commun. (PIMRC'07) (, Athens, Greece, 2007), pp. 1-5

17. M. Ling-Ling, S. Xiu-jie, An Improved Algorithm of Modulation Classification For Digital Communication Signals Based on Wavelet Transform. Proc. IEEE Intl. Conf. Wavelet Analysis and Pattern Recogn. (ICWAPR'07) (Beijing, China, 2007), pp. 1226-1231. vol. 3

18. P. Cheol-Sun, C. Jun-Ho, N. Sun-Phil, J. Won, Automatic Modulation Recognition of Digital Signals using Wavelet Features and SVM, in Proc. Inl Conf. Adv. Comm. Tech. (ICACT'08). Gangwon-Do, China 1, 387-390 (2008)

19. H. Ketterer, F. Jondral, A.H. Costa, Classification of modulation modes using time-frequency methods. Proc. Proc. IEEE Internatinal Conference on Acoustics, Speech and Signal Processing (ICASSP'99), vol. 5 (Phoenix Arizona, 1999), pp. 2471-2474

20. M.L.D. Wong, A.K. Nandi, Automatic digital modulation recognition using artificial neural network and genetic algorithm. Signal Process. 84(2), 351-365 (2004)

21. S. Theodoridis, K. Koutroumbas, Pattern Recognition, 4th edn. (Elsevier Inc., Canada, 2009)

22. B. Le, T. Rondeau, D. Maldonado, Modulation identification using neural networks for cognitive radios, in Proceedings of the SDR Technical Conference (SDR'05) (Phoenix, AZ, 2005)

23. O.A. Dobre, A. Abdi, Y. Bar-Ness, W. Su, Survey of automatic modulation classification techniques: classical approaches and new trends. IET Commun. 1(2), 137-156 (2007)

24. J.E. Giesbrecht, R. Clarke, D. Abbott, Modulation recognition for real HF signals. Proc. SPIE Int. Symp. Microelectr., MEMS, and Nanotech. (2006) (Brisbane, Australia, 2006), pp. 458-469. Vol. 60

25. J. Giesbrecht, R. Clarke, D. Abbott, Parameters for automatic modulation recognition of HF signals, in 10th IET International Conference on lonospheric Radio Systems and Techniques IRST 2006 (2006)

26. J. Giesbrecht, Entropic distance for automatic modulation recognition of HF signals, in 11th IET International Conference on lonospheric Radio Systems and Techniques IRST 2009 (Edinburgh, IET, UK, 2009)
27. H. Alharbi, S. Mobien, S. Alshebeili, F. Alturki, Classification of Digitally Modulated Signals in Presence of Non-Gaussian HF Noise, in Proc. 7th Intl. Symp. Wireless Commun. Syst. (ISWCS'10) (, York, United Kingdom, 2010), pp. 815-819

28. H. Alharbi, S. Mobien, S. Alshebeili, F. Alturki, Robustness of digitally modulated signal features against variation in HF noise model. EURASIP J. Wirel. Commun. Netw. 2011, 24 (2011)

29. R. De Gaudenzi, A. Guillen, A. Martinez, Performance analysis of turbo-coded APSK modulations over nonlinear satellite channels. IEEE Trans. Wirel. Commun. 5(9), 2396-2407 (2006)

30. P. Ciblat, M. Ghogho, Blind NLLS carrier frequency-offset estimation for QAM, PSK, and PAM modulations: performance at low SNR. IEEE Trans. Commun. 54(10), 1725-1730 (2006)

31. F. Xie, C. Li, G. Wan, An Efficient and Simple Method of MPSK Modulation Classification, 4th International Conf. on Wireless Communications, Networking and Mobile Computing (WiCOM '08) (Dilian, China, 2008), pp. 1-3

32. J.G. Proakis, Digital Communications (McGraw-Hill, New York, 2001)

33. P. Seungkeun, C. Heechang, N. Sangsin, Intersymbol interference due to mismatched roll-off factors and sampling jitter over a Gaussian channel. Electron. Lett. 33(24), 2016-2017 (1997)

34. S.D. Assimonis, M. Matthaiou, G.K. Karagiannidis, J.A. Nossek, Improved parametric families of intersymbol interference-free Nyquist pulses using inner and outer functions. IET Signal Process. 5(2), 157-163 (2011)

35. F.M. Gardner, A BPSK/QPSK timing-error detector for sampled receivers. IEEE Trans. Commun. 34(5), 423-429 (1986)

36. M.A. Rahman, C.S. Sum, R. Funada, T. Baykas, J. Wang, S. Sasaki, H. Harada, S. Kato, Error rate analysis of band-limited BPSK with Nakagami/Nakagami ACl considering nonlinear amplifier and diversity. IEEE Trans. Veh. Technol. 59(3), 1523-1529 (2010)

37. M. Leconte, M. Testard, "A model of high frequencies (H.F) channel used to design a modem of 9600 bits/s rate in 3 kHz of bandwidth," MILCOM 97 Proceedings. 1, 351-355 (1997)

38. B. Mobaseri, Constellation shape as a robust signature for digital modulation recognition, in Proc. IEEE Military Communs. Conf. (MILCOM'99), Volume 1, Issue (NJ, USA, 1999), pp. 442-446

39. N. Ahmadi, B. Reza, Modulation classification of QAM and PSK from their constellation using Genetic Algorithm and hierarchical clustering, in Proc. Intl. Conf. inf and commun. Tech.: from Theory to Appl. (ICTTA'08) (Umayyad Palace, Damascus, Syria, 2008), pp. 1-5

40. N. Ahmadi, B. Reza, A template matching approach to classification of QAM modulation using genetic algorithm. Signal Process. Int. J. 3(5), 95-109 (2009)

41. W. Kam-Tim, K. Chi-Wah, Clustering based distribution fitting algorithm for Automatic Modulation Recognition, in Proc. IEEE Symp. Comp. and Commun. (ISCC'07) (2007), pp. 13-18

42. Y. Changyi, L. Bingbing, L. Yanling, Modulation Classification of MQAM Signals from Their Constellation Using Clustering, in Proc. Intl. Conf. Commun. Software and Networks (ICCSN'10) (2010), pp. 303-306

doi:10.1186/1687-6180-2012-238

Cite this article as: Alharbi et al:: Automatic modulation classification of digital modulations in presence of HF noise. EURASIP Journal on Advances in Signal Processing 2012 2012:238.

\section{Submit your manuscript to a SpringerOpen ${ }^{\odot}$ journal and benefit from:}

- Convenient online submission

Rigorous peer review

- Immediate publication on acceptance

- Open access: articles freely available online

- High visibility within the field

- Retaining the copyright to your article

Submit your next manuscript at springeropen.com 